\title{
Some $q$-supercongruences modulo the fourth power of a cyclotomic polynomial to
}

\author{
Chuanan Wei \\ School of Biomedical Information and Engineering, \\ Hainan Medical University, Haikou 571199, China \\ weichuanan78@163.com
}

\begin{abstract}
In terms of the creative microscoping method recently introduced by Guo and Zudilin and the Chinese remainder theorem for coprime polynomials, we establish a $q$-supercongruence with two parameters modulo $[n] \Phi_{n}(q)^{3}$. Here $[n]=\left(1-q^{n}\right) /(1-q)$ and $\Phi_{n}(q)$ is the $n$-th cyclotomic polynomial in $q$. In particular, we confirm a recent conjecture of Guo and give a complete $q$-analogue of Long's supercongruence. The latter is also a generalization of a recent $q$-supercongruence obtained by Guo and Schlosser.

Keywords: basic hypergeometric series; Watson's ${ }_{8} \phi_{7}$ transformation; $q$-supercongruence AMS Subject Classifications: 33D15; 11A07; 11B65
\end{abstract}

\section{Introduction}

For a complex variable $x$, define the shifted-factorial to be

$$
(x)_{0}=1 \quad \text { and } \quad(x)_{n}=x(x+1) \cdots(x+n-1) \quad \text { when } n \in \mathbb{N} .
$$

In his second letter to Hardy on February 27, 1913, Ramanujan mentioned the identity

$$
\sum_{k=0}^{\infty}(-1)^{k}(4 k+1) \frac{\left(\frac{1}{2}\right)_{k}^{5}}{k !^{5}}=\frac{2}{\Gamma\left(\frac{3}{4}\right)^{4}},
$$

where $\Gamma(x)$ is the Gamma function. In 1997, Van Hamme [21, (A.2)] conjectured that (1.1) possesses the nice $p$-adic analogue:

$$
\sum_{k=0}^{(p-1) / 2}(-1)^{k}(4 k+1) \frac{\left(\frac{1}{2}\right)_{k}^{5}}{k !^{5}} \equiv\left\{\begin{array}{lll}
-\frac{p}{\Gamma_{p}\left(\frac{3}{4}\right)^{4}}\left(\bmod p^{3}\right), & \text { if } p \equiv 1 \quad(\bmod 4), \\
0 \quad\left(\bmod p^{3}\right), & \text { if } p \equiv 3 \quad(\bmod 4) .
\end{array}\right.
$$

Here and throughout the paper, $p$ always denotes an odd prime and $\Gamma_{p}(x)$ is the $p$ adic Gamma function. The supercongruence (1.2) was later proved by McCarthy and Osburn [17]. In 2015, Swisher [19] showed that (1.2) also holds modulo $p^{5}$ for $p \equiv 1$

\footnotetext{
${ }^{*}$ This work is supported by the National Natural Science Foundation of China (No. 11661032).
} 
$(\bmod 4)$ and $p>5$. Recently, Liu [14] found another partial generalization of it: for $p \equiv 3$ $(\bmod 4)$ and $p>3$,

$$
\sum_{k=0}^{(p-1) / 2}(-1)^{k}(4 k+1) \frac{\left(\frac{1}{2}\right)_{k}^{5}}{k !^{5}} \equiv-\frac{p^{3}}{16} \Gamma_{p}\left(\frac{1}{4}\right)^{4} \quad\left(\bmod p^{4}\right)
$$

It is known that some of the truncated hypergeometric series are related to the number of rational points on certain algebraic varieties over finite fields and further to coefficients of modular forms. For example, on the basis of the result of Ahlgren and Ono in [1], Kilbourn [2] proved Van Hammes (M.2) supercongruence:

$$
\sum_{k=0}^{(p-1) / 2} \frac{\left(\frac{1}{2}\right)_{k}^{4}}{k !^{4}} \equiv a_{p} \quad\left(\bmod p^{3}\right)
$$

where $a_{p}$ is the $p$-th coefficient of a weight 4 modular form

$$
\eta(2 z)^{4} \eta(4 z)^{4}:=q \prod_{n=1}^{\infty}\left(1-q^{2 n}\right)^{4}\left(1-q^{4 n}\right)^{4}, \quad q=e^{2 i \pi z} .
$$

In 2011, Long [16] obtained the following two supercongruences:

$$
\begin{aligned}
& \sum_{k=0}^{(p-1) / 2}(4 k+1) \frac{\left(\frac{1}{2}\right)_{k}^{4}}{k !^{4}} \equiv p \quad\left(\bmod p^{4}\right) \\
& \sum_{k=0}^{(p-1) / 2}(4 k+1) \frac{\left(\frac{1}{2}\right)_{k}^{6}}{k !^{6}} \equiv p \sum_{k=0}^{(p-1) / 2} \frac{\left(\frac{1}{2}\right)_{k}^{4}}{k !^{4}} \quad\left(\bmod p^{4}\right),
\end{aligned}
$$

where $p>3$. According to (1.4), the supercongruence (1.6) can be written as

$$
\sum_{k=0}^{(p-1) / 2}(4 k+1) \frac{\left(\frac{1}{2}\right)_{k}^{6}}{k !^{6}} \equiv p a_{p} \quad\left(\bmod p^{4}\right) \quad \text { for } p>3 .
$$

For two complex numbers $x$ and $q$, define the $q$-shifted factorial to be

$$
(x ; q)_{0}=1 \quad \text { and } \quad(x ; q)_{n}=(1-x)(1-x q) \cdots\left(1-x q^{n-1}\right) \quad \text { when } n \in \mathbb{N} .
$$

For shortening many of the formulas in this paper, we adopt the notation

$$
\left(x_{1}, x_{2}, \ldots, x_{r} ; q\right)_{n}=\left(x_{1} ; q\right)_{n}\left(x_{2} ; q\right)_{n} \cdots\left(x_{r} ; q\right)_{n} .
$$

Following Gasper and Rahman [3], define the basic hypergeometric series by

$$
{ }_{r} \phi_{s}\left[\begin{array}{c}
a_{1}, a_{2}, \ldots, a_{r} \\
b_{1}, b_{2}, \ldots, b_{s}
\end{array} ; q, z\right]=\sum_{k=0}^{\infty} \frac{\left(a_{1}, a_{2}, \ldots, a_{r} ; q\right)_{k}}{\left(q, b_{1}, b_{2}, \ldots, b_{s} ; q\right)_{k}}\left\{(-1)^{k} q^{\left(\begin{array}{c}
k \\
2
\end{array}\right)}\right\}^{1+s-r} z^{k}
$$


Then the $q$-Chu-Vandermonde sum (cf. [3, Appendix (II.6)]), a terminating $q$-analogue of Whipple's ${ }_{3} F_{2}$ sum (cf. [3, Appendix (II.19)]) and Watson's ${ }_{8} \phi_{7}$ transformation (cf. [3, Appendix (III.18)]) can be expressed as follows:

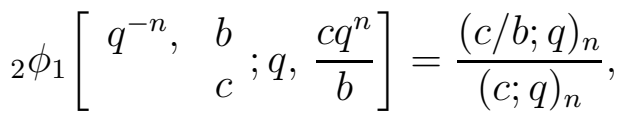

$$
\begin{aligned}
& { }_{4} \phi_{3}\left[\begin{array}{cccc}
q^{-n}, & q^{1+n}, & b, & -b \\
c, & b^{2} q / c, & -q
\end{array} ; q, q\right]=q^{\left(\begin{array}{c}
1+n \\
2
\end{array}\right)} \frac{\left(b^{2} q^{1-n} / c, c q^{-n} ; q^{2}\right)_{n}}{\left(b^{2} q / c, c ; q\right)_{n}},
\end{aligned}
$$

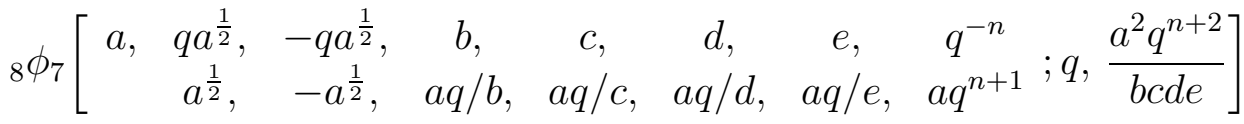

$$
\begin{aligned}
& =\frac{(a q, a q / d e ; q)_{n}}{(a q / d, a q / e ; q)_{n}}{ }_{4} \phi_{3}\left[\begin{array}{c}
a q / b c, d, e, q^{-n} \\
a q / b, a q / c, d e q^{-n} / a
\end{array} ; q, q\right] .
\end{aligned}
$$

Recently, Guo [8] proved that, for any positive odd integer $n \equiv 3(\bmod 4)$,

$$
\sum_{k=0}^{(n-1) / 2} \frac{\left(q ; q^{2}\right)_{k}^{2}\left(q^{2} ; q^{4}\right)_{k}}{\left(q^{2} ; q^{2}\right)_{k}^{2}\left(q^{4} ; q^{4}\right)_{k}} q^{2 k} \equiv[n] \frac{\left(q^{3} ; q^{4}\right)_{(n-1) / 2}}{\left(q^{5} ; q^{4}\right)_{(n-1) / 2}} \quad\left(\bmod \Phi_{n}(q)^{3}\right)
$$

and proposed the following conjecture: for any positive odd integer $n \equiv 3(\bmod 4)$,

$$
\sum_{k=0}^{M}(-1)^{k}[4 k+1] \frac{\left(q ; q^{2}\right)_{k}^{4}\left(q^{2} ; q^{4}\right)_{k}}{\left(q^{2} ; q^{2}\right)_{k}^{4}\left(q^{4} ; q^{4}\right)_{k}} q^{k} \equiv[n]^{2} q^{(1+n) / 2} \frac{\left(q^{3} ; q^{4}\right)_{(n-1) / 2}}{\left(q^{5} ; q^{4}\right)_{(n-1) / 2}} \quad\left(\bmod [n] \Phi_{n}(q)^{3}\right),
$$

which is a $q$-analogue of (1.3). Here and throughout the paper, $M$ is always equal to $(n-1) / 2$ or $(n-1)$. Some different works can be stated as follows. Guo and Zudilin [12, Theorem 4.2] found the formula with two parameters: for a positive odd integer $n$,

$$
\begin{aligned}
& \sum_{k=0}^{M}[4 k+1] \frac{\left(a q, q / a, q / c, q ; q^{2}\right)_{k}}{\left(q^{2} / a, a q^{2}, c q^{2}, q^{2} ; q^{2}\right)_{k}} c^{k} \\
& \equiv[n] \frac{(c / q)^{(n-1) / 2}\left(q^{2} / c ; q^{2}\right)_{(n-1) / 2}}{\left(c q^{2} ; q^{2}\right)_{(n-1) / 2}}\left(\bmod [n]\left(1-a q^{n}\right)\left(a-q^{n}\right)\right) .
\end{aligned}
$$

Guo and Wang [11] achieved a $q$-analogue of (1.5): for any positive odd integer $n$,

$$
\sum_{k=0}^{M}[4 k+1] \frac{\left(q ; q^{2}\right)_{k}^{4}}{\left(q^{2} ; q^{2}\right)_{k}^{4}} \equiv[n] q^{(1-n) / 2}+[n]^{3} q^{(1-n) / 2} \frac{\left(n^{2}-1\right)(1-q)^{2}}{24} \quad\left(\bmod [n] \Phi_{n}(q)^{3}\right) \text {. }
$$


Guo and Schlosser [10, Theorems 2.1 and 2.2]) proved that, for a positive odd integer $n$,

$$
\begin{aligned}
& \sum_{k=0}^{M}[4 k+1] \frac{\left(q ; q^{2}\right)_{k}^{6}}{\left(q^{2} ; q^{2}\right)_{k}^{6}} q^{k} \equiv[n] q^{(1-n) / 2} \sum_{k=0}^{(n-1) / 2} \frac{\left(q ; q^{2}\right)_{k}^{4}}{\left(q^{2} ; q^{2}\right)_{k}^{4}} q^{2 k} \quad\left(\bmod [n] \Phi_{n}(q)^{2}\right), \\
& \sum_{k=0}^{M}(-1)^{k}[4 k+1] \frac{\left(q ; q^{2}\right)_{k}^{5}}{\left(q^{2} ; q^{2}\right)_{k}^{5}} q^{k^{2}+k} \equiv[n] q^{(1-n) / 2} \sum_{k=0}^{(n-1) / 2} \frac{\left(q ; q^{2}\right)_{k}^{3}}{\left(q^{2} ; q^{2}\right)_{k}^{3}} q^{2 k}\left(\bmod [n] \Phi_{n}(q)^{2}\right) .
\end{aligned}
$$

The $q$-supercongruence (1.14) is a $q$-analogue of (1.6), where the modulo $p^{4}$ condition is replaced by the weaker condition modulo $p^{3}$. An indeed $q$-analogue of (1.2) (cf. [7] and [22]) can be expressed as follows: for any positive odd integer $n$,

$$
\begin{aligned}
& \sum_{k=0}^{M}(-1)^{k}[4 k+1] \frac{\left(q ; q^{2}\right)_{k}^{4}\left(q^{2} ; q^{4}\right)_{k}}{\left(q^{2} ; q^{2}\right)_{k}^{4}\left(q^{4} ; q^{4}\right)_{k}} q^{k} \\
& \equiv\left\{\begin{array}{lll}
{[n] \frac{\left(q^{2} ; q^{4}\right)_{(n-1) / 4}^{2}}{\left(q^{4} ; q^{4}\right)_{(n-1) / 4}^{2}}\left(\bmod [n] \Phi_{n}(q)^{2}\right),} & \text { if } n \equiv 1 & (\bmod 4), \\
0 \quad\left(\bmod [n] \Phi_{n}(q)^{2}\right), & \text { if } n \equiv 3 & (\bmod 4)
\end{array}\right.
\end{aligned}
$$

We point out that more $q$-analogues of supercongruences can be found in $[5,6,19,15,18$, 20,23, 24] with various techniques.

Inspired by these work just mentioned, we shall establish the following theorem.

Theorem 1.1. Let $n$ be a positive odd integer. Then

$$
\begin{aligned}
& \sum_{k=0}^{M}[4 k+1] \frac{\left(q ; q^{2}\right)_{k}^{4}\left(c q, d q ; q^{2}\right)_{k}}{\left(q^{2} ; q^{2}\right)_{k}^{4}\left(q^{2} / c, q^{2} / d ; q^{2}\right)_{k}}\left(\frac{q}{c d}\right)^{k} \\
& \equiv\left\{[n] q^{(1-n) / 2}+[n]^{3} q^{(1-n) / 2} \frac{\left(n^{2}-1\right)(1-q)^{2}}{24}\right\} \\
& \quad \times \sum_{k=0}^{(n-1) / 2} \frac{\left(q ; q^{2}\right)_{k}^{3}\left(q / c d ; q^{2}\right)_{k}}{\left(q^{2} ; q^{2}\right)_{k}^{2}\left(q^{2} / c, q^{2} / d ; q^{2}\right)_{k}} q^{2 k}\left(\bmod [n] \Phi_{n}(q)^{3}\right) .
\end{aligned}
$$

When $c d=q$, the $q$-supercognruence (1.17) reduces to (1.13). When the parameters $c$ and $d$ are further specified, we can confirm Guo's conjecture (1.11), a $q$-analogue of (1.6), five new $q$-analogues of (1.5), and some other conclusions from this theorem.

The rest of the paper is arranged as follows. We shall display several concrete $q$ supercongruences from Theorem 1.1 in Section 2. Via the creative microscoping method, a $q$-supercongruence with four parameters modulo $\Phi_{n}(q)\left(1-a q^{n}\right)\left(a-q^{n}\right)$, which includes (1.12) and (1.14) $-(1.16)$ as special cases, will be derived in Section 3. Then we utilize it and the Chinese remainder theorem for coprime polynomials to deduce a $q$-supercongruence with three parameters modulo $\Phi_{n}(q)^{2}\left(1-a q^{n}\right)\left(a-q^{n}\right)$ and prove Theorem 1.1 in Section 4. 


\section{Concrete $q$-supergruences from Theorem 1.1}

Nine $q$-supercongruences modulo $[n] \Phi_{n}(q)^{3}$ from Theorem 1.1 will be laid out. Above all, we give the following lemma.

Lemma 2.1. Let $n$ be a positive odd integer. Then

$$
[n]^{2} \frac{\left(q^{3} ; q^{4}\right)_{(n-1) / 2}}{\left(q^{5} ; q^{4}\right)_{(n-1) / 2}} \equiv 0 \quad(\bmod [n]) .
$$

Proof. For two nonnegative integer $s, t$ with $s \leq t$, it is well known that the $q$-binomial coefficient $\left[\begin{array}{l}t \\ s\end{array}\right]$ is a polynomial in $q$ and

$$
\frac{\left(q ; q^{2}\right)_{t}}{\left(q^{2} ; q^{2}\right)_{t}}=\frac{1}{(-q ; q)_{t}^{2}}\left[\begin{array}{c}
2 t \\
t
\end{array}\right]
$$

By specifying the parameters in (11.8), Guo [8] discovered the identity

$$
{ }_{4} \phi_{3}\left[\begin{array}{cccc}
q^{1-n}, & q^{1+n}, & q, & -q \\
& q^{2+n}, & q^{2-n}, & -q^{2}
\end{array} ; q^{2}, q^{2}\right]=[n] \frac{\left(q^{3} ; q^{4}\right)_{(n-1) / 2}}{\left(q^{5} ; q^{4}\right)_{(n-1) / 2}} .
$$

For proving Lemma 2.1, it is sufficient to show that

$$
[n] \frac{\left(q^{1-n}, q^{1+n} ; q^{2}\right)_{k}}{\left(q^{2-n}, q^{2+n} ; q^{2}\right)_{k}} \equiv 0 \quad(\bmod [n]),
$$

where $0 \leq k \leq(n-1) / 2$. Through (1.7), we have

$$
\begin{aligned}
{[n] \frac{\left(q^{1-n}, q^{1+n} ; q^{2}\right)_{k}}{\left(q^{2-n}, q^{2+n} ; q^{2}\right)_{k}}=} & q^{-k}[n]^{2} \frac{\left(q ; q^{2}\right)_{(n-1) / 2-k}}{\left(q^{2} ; q^{2}\right)_{(n-1) / 2-k}} \frac{\left(q^{2} ; q^{2}\right)_{(n-1) / 2+k}}{\left(q^{3} ; q^{2}\right)_{(n-1) / 2+k}} \\
= & \frac{\left(q ; q^{2}\right)_{(n-1) / 2-k}}{\left(q^{2} ; q^{2}\right)_{(n-1) / 2-k}} \\
& \times \sum_{j=0}^{(n-1) / 2+k}(-1)^{j} q^{j^{2}+j-k}\left[\begin{array}{c}
(n-1) / 2+k \\
j
\end{array}\right]_{q^{2}} \frac{[n]^{2}}{[1+2 j]}
\end{aligned}
$$

Thus we verify the correctness of (2.2). This finishes the proof of Lemma 2.1 .

It is easy to understand that the factor $\left(q^{2} / c, q^{2} / d ; q^{2}\right)_{M}$ in the denominator of the left-hand side of (1.17) is relatively prime to $\Phi_{n}(q)$ as $c \rightarrow 1, d \rightarrow-1$ (some similar discussion will be omitted in the rest of the paper). Choosing $c=1, d=-1$ in Theorem 1.1, we obtain

$$
\sum_{k=0}^{M}(-1)^{k}[4 k+1] \frac{\left(q ; q^{2}\right)_{k}^{4}\left(q^{2} ; q^{4}\right)_{k}}{\left(q^{2} ; q^{2}\right)_{k}^{4}\left(q^{4} ; q^{4}\right)_{k}} q^{k} \equiv[n] q^{(1-n) / 2}\left\{1+[n]^{2} \frac{\left(n^{2}-1\right)(1-q)^{2}}{24}\right\}
$$




$$
\times \sum_{k=0}^{(n-1) / 2} \frac{\left(q ; q^{2}\right)_{k}^{2}\left(q^{2} ; q^{4}\right)_{k}}{\left(q^{2} ; q^{2}\right)_{k}^{2}\left(q^{4} ; q^{4}\right)_{k}} q^{2 k} \quad\left(\bmod [n] \Phi_{n}(q)^{3}\right)
$$

By means of Lemma 2.1, (1.10) and the last relation, we get the formula: for a positive odd integer $n \equiv 3(\bmod 4)$,

$$
\begin{aligned}
\sum_{k=0}^{M}(-1)^{k}[4 k+1] \frac{\left(q ; q^{2}\right)_{k}^{4}\left(q^{2} ; q^{4}\right)_{k}}{\left(q^{2} ; q^{2}\right)_{k}^{4}\left(q^{4} ; q^{4}\right)_{k}} q^{k} \equiv & {[n]^{2} q^{(1-n) / 2}\left\{1+[n]^{2} \frac{\left(n^{2}-1\right)(1-q)^{2}}{24}\right\} } \\
& \times \frac{\left(q^{3} ; q^{4}\right)_{(n-1) / 2}}{\left(q^{5} ; q^{4}\right)_{(n-1) / 2}}\left(\bmod [n] \Phi_{n}(q)^{3}\right) .
\end{aligned}
$$

It is routine to verify that

$$
\begin{gathered}
{[n]^{2} q^{(1-n) / 2}\left\{1+[n]^{2} \frac{\left(n^{2}-1\right)(1-q)^{2}}{24}\right\} \frac{\left(q^{3} ; q^{4}\right)_{(n-1) / 2}}{\left(q^{5} ; q^{4}\right)_{(n-1) / 2}}} \\
\equiv[n]^{2} q^{(1+n) / 2} \frac{\left(q^{3} ; q^{4}\right)_{(n-1) / 2}}{\left(q^{5} ; q^{4}\right)_{(n-1) / 2}} \quad\left(\bmod [n] \Phi_{n}(q)^{3}\right) .
\end{gathered}
$$

The combination of (2.3) and (2.4) confirms Guo's conjecture (1.11).

Fixing $c=d=1$ in Theorem 1.1, we achieve the $q$-analogue of (1.6).

Corollary 2.2. Let $n$ be a positive odd integer. Then

$$
\begin{aligned}
\sum_{k=0}^{M}[4 k+1] \frac{\left(q ; q^{2}\right)_{k}^{6}}{\left(q^{2} ; q^{2}\right)_{k}^{6}} q^{k} \equiv & {[n] q^{(1-n) / 2}\left\{1+[n]^{2} \frac{\left(n^{2}-1\right)(1-q)^{2}}{24}\right\} } \\
& \times \sum_{k=0}^{(n-1) / 2} \frac{\left(q ; q^{2}\right)_{k}^{4}}{\left(q^{2} ; q^{2}\right)_{k}^{4}} q^{2 k} \quad\left(\bmod [n] \Phi_{n}(q)^{3}\right) .
\end{aligned}
$$

Setting $c=d=-1$ in Theorem 1.1, we gain the first new $q$-analogue of (1.5).

Corollary 2.3. Let $n$ be a positive odd integer. Then

$$
\begin{aligned}
\sum_{k=0}^{M}[4 k+1] \frac{\left(q ; q^{2}\right)_{k}^{2}\left(q^{2} ; q^{4}\right)_{k}^{2}}{\left(q^{2} ; q^{2}\right)_{k}^{2}\left(q^{4} ; q^{4}\right)_{k}^{2}} q^{k} \equiv & {[n] q^{(1-n) / 2}\left\{1+[n]^{2} \frac{\left(n^{2}-1\right)(1-q)^{2}}{24}\right\} } \\
& \times \sum_{k=0}^{(n-1) / 2} \frac{\left(q ; q^{2}\right)_{k}^{4}}{\left(q^{4} ; q^{4}\right)_{k}^{2}} q^{2 k} \quad\left(\bmod [n] \Phi_{n}(q)^{3}\right) .
\end{aligned}
$$

Letting $c=-1, d \rightarrow \infty$ in Theorem 1.1, we obtain the second new $q$-analogue of (1.5). 
Corollary 2.4. Let $n$ be a positive odd integer. Then

$$
\begin{gathered}
\sum_{k=0}^{M}[4 k+1] \frac{\left(q ; q^{2}\right)_{k}^{3}\left(q^{2} ; q^{4}\right)_{k}}{\left(q^{2} ; q^{2}\right)_{k}^{3}\left(q^{4} ; q^{4}\right)_{k}} q^{k^{2}+k} \equiv[n] q^{(1-n) / 2}\left\{1+[n]^{2} \frac{\left(n^{2}-1\right)(1-q)^{2}}{24}\right\} \\
\times \sum_{k=0}^{(n-1) / 2} \frac{\left(q ; q^{2}\right)_{k}^{3}}{\left(q^{2} ; q^{2}\right)_{k}\left(q^{4} ; q^{4}\right)_{k}} q^{2 k} \quad\left(\bmod [n] \Phi_{n}(q)^{3}\right) .
\end{gathered}
$$

Letting $c=-1, d \rightarrow 0$ in Theorem 1.1, we get the third new $q$-analogue of (1.5).

Corollary 2.5. Let $n$ be a positive odd integer. Then

$$
\begin{aligned}
\sum_{k=0}^{M}[4 k+1] \frac{\left(q ; q^{2}\right)_{k}^{3}\left(q^{2} ; q^{4}\right)_{k}}{\left(q^{2} ; q^{2}\right)_{k}^{3}\left(q^{4} ; q^{4}\right)_{k}} q^{-k^{2}} \equiv & {[n] q^{(1-n) / 2}\left\{1+[n]^{2} \frac{\left(n^{2}-1\right)(1-q)^{2}}{24}\right\} } \\
& \times \sum_{k=0}^{(n-1) / 2} \frac{\left(q ; q^{2}\right)_{k}^{3}}{\left(q^{2} ; q^{2}\right)_{k}\left(q^{4} ; q^{4}\right)_{k}}(-q)^{k} \quad\left(\bmod [n] \Phi_{n}(q)^{3}\right) .
\end{aligned}
$$

Letting $c \rightarrow \infty, d \rightarrow \infty$ in Theorem 1.1, we are led to the fourth new $q$-analogue of (1.5).

Corollary 2.6. Let $n$ be a positive odd integer. Then

$$
\begin{aligned}
\sum_{k=0}^{M}[4 k+1] \frac{\left(q ; q^{2}\right)_{k}^{4}}{\left(q^{2} ; q^{2}\right)_{k}^{4}} q^{2 k^{2}+k} \equiv & {[n] q^{(1-n) / 2}\left\{1+[n]^{2} \frac{\left(n^{2}-1\right)(1-q)^{2}}{24}\right\} } \\
& \times \sum_{k=0}^{(n-1) / 2} \frac{\left(q ; q^{2}\right)_{k}^{3}}{\left(q^{2} ; q^{2}\right)_{k}^{2}} q^{2 k}\left(\bmod [n] \Phi_{n}(q)^{3}\right)
\end{aligned}
$$

Letting $c \rightarrow 0, d \rightarrow 0$ in Theorem 1.1, we arrive at the fifth new $q$-analogue of (1.5).

Corollary 2.7. Let $n$ be a positive odd integer. Then

$$
\begin{aligned}
\sum_{k=0}^{M}[4 k+1] \frac{\left(q ; q^{2}\right)_{k}^{4}}{\left(q^{2} ; q^{2}\right)_{k}^{4}} q^{-2 k^{2}-k} \equiv & {[n] q^{(1-n) / 2}\left\{1+[n]^{2} \frac{\left(n^{2}-1\right)(1-q)^{2}}{24}\right\} } \\
& \times \sum_{k=0}^{(n-1) / 2} \frac{\left(q ; q^{2}\right)_{k}^{3}}{\left(q^{2} ; q^{2}\right)_{k}^{2}}(-q)^{-k^{2}}\left(\bmod [n] \Phi_{n}(q)^{3}\right) .
\end{aligned}
$$

The case $c=d=q^{-2}$ of Theorem 1.1 yields the following result. 
Corollary 2.8. Let $n$ be a positive odd integer. Then

$$
\begin{aligned}
\sum_{k=0}^{M}[4 k+1] \frac{\left(q ; q^{2}\right)_{k}^{4}\left(q^{-1} ; q^{2}\right)_{k}^{2}}{\left(q^{2} ; q^{2}\right)_{k}^{4}\left(q^{4} ; q^{2}\right)_{k}^{2}} q^{5 k} \equiv & {[n] q^{(1-n) / 2}\left\{1+[n]^{2} \frac{\left(n^{2}-1\right)(1-q)^{2}}{24}\right\} } \\
& \times \sum_{k=0}^{(n-1) / 2} \frac{\left(q ; q^{2}\right)_{k}^{3}\left(q^{5} ; q^{2}\right)_{k}}{\left(q^{2} ; q^{2}\right)_{k}^{2}\left(q^{4} ; q^{2}\right)_{k}^{2}} q^{2 k} \quad\left(\bmod [n] \Phi_{n}(q)^{3}\right)
\end{aligned}
$$

Choosing $M=\left(p^{r}-1\right) / 2$ and letting $q \rightarrow 1$ in the above $q$-supercongruence, we obtain

$$
\sum_{k=0}^{\left(p^{r}-1\right) / 2}(4 k+1) \frac{\left(\frac{1}{2}\right)_{k}^{4}\left(-\frac{1}{2}\right)_{k}^{2}}{k !^{4}(k+1) !^{2}} \equiv p^{r} \sum_{k=0}^{\left(p^{r}-1\right) / 2} \frac{\left(\frac{1}{2}\right)_{k}^{3}\left(\frac{5}{2}\right)_{k}}{k !^{2}(k+1) !^{2}} \quad\left(\bmod p^{r+3}\right) \quad \text { for } p>3
$$

The case $c=q^{-2}, d=1$ of Theorem 1.1 is the following result.

Corollary 2.9. Let $n$ be a positive odd integer. Then

$$
\begin{aligned}
\sum_{k=0}^{M}[4 k+1] \frac{\left(q ; q^{2}\right)_{k}^{5}\left(q^{-1} ; q^{2}\right)_{k}}{\left(q^{2} ; q^{2}\right)_{k}^{5}\left(q^{4} ; q^{2}\right)_{k}} q^{3 k} \equiv & {[n] q^{(1-n) / 2}\left\{1+[n]^{2} \frac{\left(n^{2}-1\right)(1-q)^{2}}{24}\right\} } \\
& \times \sum_{k=0}^{(n-1) / 2} \frac{\left(q ; q^{2}\right)_{k}^{3}\left(q^{3} ; q^{2}\right)_{k}}{\left(q^{2} ; q^{2}\right)_{k}^{3}\left(q^{4} ; q^{2}\right)_{k}} \quad\left(\bmod [n] \Phi_{n}(q)^{3}\right)
\end{aligned}
$$

Fixing $M=\left(p^{r}-1\right) / 2$ and letting $q \rightarrow 1$ in the above $q$-supercognruence, we get

$$
\sum_{k=0}^{\left(p^{r}-1\right) / 2}(4 k+1) \frac{\left(\frac{1}{2}\right)_{k}^{5}\left(-\frac{1}{2}\right)_{k}}{k ! 5(k+1) !} \equiv p^{r} \sum_{k=0}^{\left(p^{r}-1\right) / 2} \frac{\left(\frac{1}{2}\right)_{k}^{3}\left(\frac{3}{2}\right)_{k}}{k !^{3}(k+1) !} \quad\left(\bmod p^{r+3}\right) \quad \text { for } p>3
$$

\section{A q-supergruence with four parematers modulo $\Phi_{n}(q)\left(1-a q^{n}\right)\left(a-q^{n}\right)$}

In this section, we shall deduce a $q$-supercongruence with four parameters modulo $\Phi_{n}(q)(1-$ $\left.a q^{n}\right)\left(a-q^{n}\right)$ in terms of the creative telescoping method. When the parameters are specified, it can produce (1.12) and (1.14) $-(1.16)$.

We first require the following lemma (see [10, Lemma 3.1]).

Lemma 3.1. Let $n$ be a positive odd integer and $k$ an integer with $0 \leq k \leq(n-1) / 2$. Then

$$
\frac{\left(x q ; q^{2}\right)_{(n-1) / 2-k}}{\left(q^{2} / x ; q^{2}\right)_{(n-1) / 2-k}} \equiv(-x)^{(n-1) / 2-2 k} \frac{\left(x q ; q^{2}\right)_{k}}{\left(q^{2} / x ; q^{2}\right)_{k}} q^{(n-1)^{2} / 4+k} \quad\left(\bmod \Phi_{n}(q)\right) .
$$

We also need two more lemmas. 
Lemma 3.2. Let $n$ be a positive odd integer. Then

$$
\sum_{k=0}^{M}[4 k+1] \frac{\left(a q, q / a, q / b, c q, d q, q ; q^{2}\right)_{k}}{\left(q^{2} / a, a q^{2}, b q^{2}, q^{2} / c, q^{2} / d, q^{2} ; q^{2}\right)_{k}}\left(\frac{b q}{c d}\right)^{k} \equiv 0 \quad\left(\bmod \Phi_{n}(q)\right) .
$$

Proof. Let $\alpha_{q}(k)$ denote the $k$-th term on the left-hand side of (3.1), i.e.,

$$
\alpha_{q}(k)=[4 k+1] \frac{\left(a q, q / a, q / b, c q, d q, q ; q^{2}\right)_{k}}{\left(q^{2} / a, a q^{2}, b q^{2}, q^{2} / c, q^{2} / d, q^{2} ; q^{2}\right)_{k}}\left(\frac{b q}{c d}\right)^{k} .
$$

According to Lemma 3.1, we obtain

$$
\alpha_{q}((n-1) / 2-k) \equiv-\alpha_{q}(k) \quad\left(\bmod \Phi_{n}(q)\right) .
$$

When $(n-1) / 2$ is odd, it is not difficult to verify that

$$
\sum_{k=0}^{(n-1) / 2}[4 k+1] \frac{\left(a q, q / a, q / b, c q, d q, q ; q^{2}\right)_{k}}{\left(q^{2} / a, a q^{2}, b q^{2}, q^{2} / c, q^{2} / d, q^{2} ; q^{2}\right)_{k}}\left(\frac{b q}{c d}\right)^{k} \equiv 0 \quad\left(\bmod \Phi_{n}(q)\right) .
$$

When $(n-1) / 2$ is even, the central term $\alpha_{q}((n-1) / 4)$ will remain. Since it has the factor $[n]$, (3.2) is also true in this instance. If $n>(n-1) / 2$, the factor $\left(1-q^{n}\right)$ appears in the numerator of $\alpha_{q}(k)$. This indicates

$$
\sum_{k=0}^{n-1}[4 k+1] \frac{\left(a q, q / a, q / b, c q, d q, q ; q^{2}\right)_{k}}{\left(q^{2} / a, a q^{2}, b q^{2}, q^{2} / c, q^{2} / d, q^{2} ; q^{2}\right)_{k}}\left(\frac{b q}{c d}\right)^{k} \equiv 0 \quad\left(\bmod \Phi_{n}(q)\right)
$$

as desired.

Lemma 3.3. Let $n$ be a positive odd integer. Then

$$
\sum_{k=0}^{M}[4 k+1] \frac{\left(a q, q / a, q / b, c q, d q, q ; q^{2}\right)_{k}}{\left(q^{2} / a, a q^{2}, b q^{2}, q^{2} / c, q^{2} / d, q^{2} ; q^{2}\right)_{k}}\left(\frac{b q}{c d}\right)^{k} \equiv 0 \quad(\bmod [n]) .
$$

Proof. For $n>1$, let $\zeta \neq 1$ be an $n$-th root of unity, which is not necessarily primitive. This means that $\zeta$ is a primitive root of unity of odd degree $m \mid n$. Lemma 3.2 with $n=m$ implies that

$$
\sum_{k=0}^{m-1} \alpha_{\zeta}(k)=\sum_{k=0}^{(m-1) / 2} \alpha_{\zeta}(k)=0
$$

By means of the relation:

$$
\frac{\alpha_{\zeta}(j m+k)}{\alpha_{\zeta}(j m)}=\lim _{q \rightarrow \zeta} \frac{\alpha_{q}(j m+k)}{\alpha_{q}(j m)}=\alpha_{\zeta}(k),
$$


we achieve

$$
\begin{gathered}
\sum_{k=0}^{n-1} \alpha_{\zeta}(k)=\sum_{j=0}^{n / m-1} \sum_{k=0}^{m-1} \alpha_{\zeta}(j m+k)=\sum_{j=0}^{n / m-1} \alpha_{\zeta}(j m) \sum_{k=0}^{m-1} \alpha_{\zeta}(k)=0, \\
\sum_{k=0}^{(n-1) / 2} \alpha_{\zeta}(k)=\sum_{j=0}^{(n / m-3) / 2} \alpha_{\zeta}(j m) \sum_{k=0}^{m-1} \alpha_{\zeta}(k)+\sum_{k=0}^{(m-1) / 2} \alpha_{\zeta}((n-m) / 2+k)=0 .
\end{gathered}
$$

The last two equations tell us that $\sum_{k=0}^{n-1} \alpha_{q}(k)$ and $\sum_{k=0}^{(n-1) / 2} \alpha_{q}(k)$ are both divisible by the cyclotomic polynomials $\Phi_{m}(q)$. Because this is correct for any divisor $m>1$ of $n$, we conclude that they are divisible by

$$
\prod_{m \mid n, m>1} \Phi_{m}(q)=[n]
$$

This completes the proof.

We now state our main result in this section.

Theorem 3.4. Let $n$ be a positive odd integer. Then

$$
\begin{aligned}
& \sum_{k=0}^{M}[4 k+1] \frac{\left(a q, q / a, q / b, c q, d q, q ; q^{2}\right)_{k}}{\left(q^{2} / a, a q^{2}, b q^{2}, q^{2} / c, q^{2} / d, q^{2} ; q^{2}\right)_{k}}\left(\frac{b q}{c d}\right)^{k} \\
& \equiv[n](b / q)^{(n-1) / 2} \frac{\left(q^{2} / b ; q^{2}\right)_{(n-1) / 2}}{\left(b q^{2} ; q^{2}\right)_{(n-1) / 2}} \\
& \quad \times \sum_{k=0}^{(n-1) / 2} \frac{\left(a q, q / a, q / b, q / c d ; q^{2}\right)_{k}}{\left(q^{2}, q^{2} / b, q^{2} / c, q^{2} / d ; q^{2}\right)_{k}} q^{2 k}\left(\bmod \Phi_{n}(q)\left(1-a q^{n}\right)\left(a-q^{n}\right)\right) .
\end{aligned}
$$

Proof. When $a=q^{-n}$ or $a=q^{n}$, the left-hand side of (3.3) is equal to

$$
\begin{aligned}
& \sum_{k=0}^{M}[4 k+1] \frac{\left(q^{1-n}, q^{1+n}, q / b, c q, d q, q ; q^{2}\right)_{k}}{\left(q^{2+n}, q^{2-n}, b q^{2}, q^{2} / c, q^{2} / d, q^{2} ; q^{2}\right)_{k}}\left(\frac{b q}{c d}\right)^{k} \\
& ={ }_{8} \phi_{7}\left[\begin{array}{cccccccc}
q, & q^{\frac{5}{2}}, & -q^{\frac{5}{2}}, & c q, & d q, & q / b, & q^{1+n}, & q^{1-n} \\
& q^{\frac{1}{2}}, & -q^{\frac{1}{2}}, & q^{2} / c, & q^{2} / d, & b q^{2}, & q^{2-n}, & q^{2+n}
\end{array} ; q^{2}, \frac{b q}{c d}\right] .
\end{aligned}
$$

Via (1.9), the right-hand side of (3.4) can be rewritten as

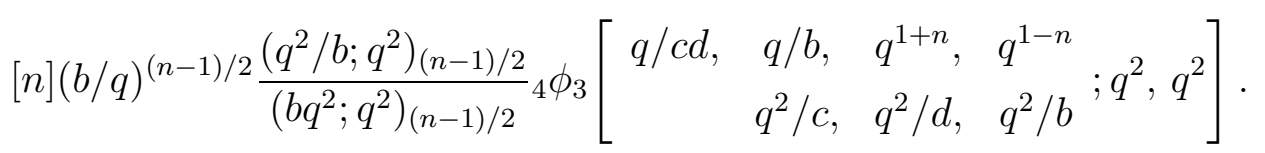

This proves that the $q$-supercongruence (3.3) holds modulo $\left(1-a q^{n}\right)$ or $\left(a-q^{n}\right)$. Lemma 3.2 implies that (3.3) is also true modulo $\Phi_{n}(q)$. Since $\Phi_{n}(q),\left(1-a q^{n}\right)$, and $\left(a-q^{n}\right)$ are pairwise relatively prime polynomials, we obtain (3.3). 
In the light of Lemma 3.3, the $q$-supercongruence (3.3) becomes (1.12) when $c d=q$. Letting $a \rightarrow 1, b=c=d=1$ in (3.3) and using (2.1) and Lemma 3.3, we get (1.14). If we take $a \rightarrow 1, b=c=1, d \rightarrow \infty$, then the $q$-supercongruence (3.3) reduces to (1.15) under (2.1) and Lemma 3.3

Moreover, letting $a \rightarrow 1$ and putting $b=c=1, d=-1$ in (3.3) and employing (2.1) and Lemma 3.3, we have

$$
\begin{aligned}
& \sum_{k=0}^{M}(-1)^{k}[4 k+1] \frac{\left(q ; q^{2}\right)_{k}^{4}\left(q^{2} ; q^{2}\right)_{k}}{\left(q^{2} ; q^{2}\right)_{k}^{4}\left(q^{4} ; q^{4}\right)_{k}} q^{k} \\
& \quad \equiv[n] q^{(1-n) / 2} \sum_{k=0}^{(n-1) / 2} \frac{\left(q ; q^{2}\right)_{k}^{2}\left(q^{2} ; q^{4}\right)_{k}}{\left(q^{2} ; q^{2}\right)_{k}^{2}\left(q^{4} ; q^{4}\right)_{k}} q^{2 k} \quad\left(\bmod [n] \Phi_{n}(q)^{2}\right)
\end{aligned}
$$

In view of the following $q$-supercongruence (see [13, Theorem 2])

$$
\sum_{k=0}^{(n-1) / 2} \frac{\left(q ; q^{2}\right)_{k}^{2}\left(q^{2} ; q^{4}\right)_{k}}{\left(q^{2} ; q^{2}\right)_{k}^{2}\left(q^{4} ; q^{4}\right)_{k}} q^{2 k} \equiv\left\{\begin{array}{lll}
\frac{\left(q^{2} ; q^{4}\right)_{(n-1) / 4}^{2}}{\left(q^{4} ; q^{4}\right)_{(n-1) / 4}^{2}} q^{(n-1) / 2}\left(\bmod \Phi_{n}(q)^{2}\right), & \text { if } n \equiv 1 & (\bmod 4) \\
0 \quad\left(\bmod \Phi_{n}(q)^{2}\right), & \text { if } n \equiv 3 & (\bmod 4)
\end{array}\right.
$$

we immediately get (1.16).

\section{Proof of Theorem 1.1}

In this section, we shall establish a $q$-supercongruence with three parameters modulo $\Phi_{n}(q)^{2}\left(1-a q^{n}\right)\left(a-q^{n}\right)$ by using Theorem 3.4 and the Chinese remainder theorem for coprime polynomials. Then we utilize it to give the proof of Theorem 1.1 .

In order to reach the goals, we need the following lemma.

Lemma 4.1. Let $n$ be a positive odd integer. Then

$$
\begin{aligned}
\sum_{k=0}^{M}[ & 4 k+1] \frac{\left(a q, q / a, q / b, c q, d q, q ; q^{2}\right)_{k}}{\left(q^{2} / a, a q^{2}, b q^{2}, q^{2} / c, q^{2} / d, q^{2} ; q^{2}\right)_{k}}\left(\frac{b q}{c d}\right)^{k} \\
\equiv[n] \frac{\left(q ; q^{2}\right)_{(n-1) / 2}^{2}}{\left(a q^{2}, q^{2} / a ; q^{2}\right)_{(n-1) / 2}} & \\
& \times \sum_{k=0}^{(n-1) / 2} \frac{\left(a q, q / a, q / b, q / c d ; q^{2}\right)_{k}}{\left(q^{2}, q^{2} / b, q^{2} / c, q^{2} / d ; q^{2}\right)_{k}} q^{2 k}\left(\bmod \left(b-q^{n}\right)\right) .
\end{aligned}
$$


Proof. When $b=q^{n}$, the left-hand side of (4.1) is equal to

$$
\begin{aligned}
& \sum_{k=0}^{M}[4 k+1] \frac{\left(a q, q / a, q^{1-n}, c q, d q, q ; q^{2}\right)_{k}}{\left(q^{2} / a, a q^{2}, q^{2+n}, q^{2} / c, q^{2} / d, q^{2} ; q^{2}\right)_{k}}\left(\frac{q^{n+1}}{c d}\right)^{k}
\end{aligned}
$$

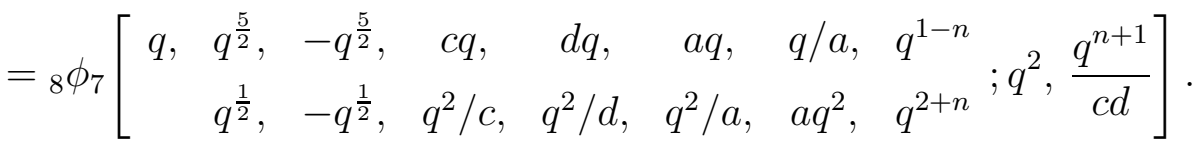

Via (1.9), the right-hand side of (4.2) can be rewritten as

$$
[n] \frac{\left(q ; q^{2}\right)_{(n-1) / 2}^{2}}{\left(a q^{2}, q^{2} / a ; q^{2}\right)_{(n-1) / 2}}{ }_{4} \phi_{3}\left[\begin{array}{rrrr}
q / c d, & a q, & q / a, & q^{1-n} \\
& q^{2} / c, & q^{2} / d, & q^{2-n}
\end{array} ; q^{2}, q^{2}\right] .
$$

This proves that Lemma 3.2 holds modulo $\left(b-q^{n}\right)$.

We now give a parametric generaliztation of Theorem 1.1 .

Theorem 4.2. Let $n$ be a positive odd integer. Then, modulo $\Phi_{n}(q)^{2}\left(1-a q^{n}\right)\left(a-q^{n}\right)$,

$$
\begin{aligned}
& \sum_{k=0}^{M}[4 k+1] \frac{\left(a q, q / a, c q, d q ; q^{2}\right)_{k}\left(q ; q^{2}\right)_{k}^{2}}{\left(q^{2} / a, a q^{2}, q^{2} / c, q^{2} / d ; q^{2}\right)_{k}\left(q^{2} ; q^{2}\right)_{k}^{2}}\left(\frac{q}{c d}\right)^{k} \\
& \equiv[n] \Omega_{q}(a, n) \sum_{k=0}^{(n-1) / 2} \frac{\left(a q, q / a, q / c d, q ; q^{2}\right)_{k}}{\left(q^{2} / c, q^{2} / d ; q^{2}\right)_{k}\left(q^{2} ; q^{2}\right)_{k}^{2}} q^{2 k}
\end{aligned}
$$

where the notation on the right-hand side denotes

$$
\Omega_{q}(a, n)=q^{(1-n) / 2}+q^{(1-n) / 2} \frac{\left(1-a q^{n}\right)\left(a-q^{n}\right)}{(1-a)^{2}}\left\{1-\frac{n(1-a) a^{(n-1) / 2}}{1-a^{n}}\right\} .
$$

Proof. It is clear that the polynomials $\left(1-a q^{n}\right)\left(a-q^{n}\right)$ and $\left(b-q^{n}\right)$ are relatively prime. Noting the $q$-congruences

$$
\begin{aligned}
\frac{\left(b-q^{n}\right)\left(a b-1-a^{2}+a q^{n}\right)}{(a-b)(1-a b)} & \equiv 1 \quad\left(\bmod \left(1-a q^{n}\right)\left(a-q^{n}\right)\right), \\
\frac{\left(1-a q^{n}\right)\left(a-q^{n}\right)}{(a-b)(1-a b)} & \equiv 1 \quad\left(\bmod \left(b-q^{n}\right)\right)
\end{aligned}
$$

and employing the Chinese remainder theorem for coprime polynomials, we can derive, from Lemmas 3.2 and 4.1 and Theorem 3.4 , the following $q$-congruence: modulo $\Phi_{n}(q)(1-$ $\left.a q^{n}\right)\left(a-q^{n}\right)\left(b-q^{n}\right)$

$$
\begin{aligned}
& \sum_{k=0}^{M}[4 k+1] \frac{\left(a q, q / a, q / b, c q, d q, q ; q^{2}\right)_{k}}{\left(q^{2} / a, a q^{2}, b q^{2}, q^{2} / c, q^{2} / d, q^{2} ; q^{2}\right)_{k}}\left(\frac{b q}{c d}\right)^{k} \\
& \quad \equiv[n] R_{q}(a, b, n) \sum_{k=0}^{(n-1) / 2} \frac{\left(a q, q / a, q / b, q / c d ; q^{2}\right)_{k}}{\left(q^{2}, q^{2} / b, q^{2} / c, q^{2} / d ; q^{2}\right)_{k}} q^{2 k}
\end{aligned}
$$


where

$$
\begin{aligned}
R_{q}(a, b, n)= & \frac{\left(b-q^{n}\right)\left(a b-1-a^{2}+a q^{n}\right)}{(a-b)(1-a b)} \frac{(b / q)^{(n-1) / 2}\left(q^{2} / b ; q^{2}\right)_{(n-1) / 2}}{\left(b q^{2} ; q^{2}\right)_{(n-1) / 2}} \\
& +\frac{\left(1-a q^{n}\right)\left(a-q^{n}\right)}{(a-b)(1-a b)} \frac{\left(q ; q^{2}\right)_{(n-1) / 2}^{2}}{\left(a q^{2}, q^{2} / a ; q^{2}\right)_{(n-1) / 2}} .
\end{aligned}
$$

Letting $b \rightarrow 1$ in (4.3), we conclude that, modulo $\Phi_{n}(q)^{2}\left(1-a q^{n}\right)\left(a-q^{n}\right)$,

$$
\begin{aligned}
& \sum_{k=0}^{M}[4 k+1] \frac{\left(a q, q / a, c q, d q ; q^{2}\right)_{k}\left(q ; q^{2}\right)_{k}^{2}}{\left(q^{2} / a, a q^{2}, q^{2} / c, q^{2} / d ; q^{2}\right)_{k}\left(q^{2} ; q^{2}\right)_{k}^{2}}\left(\frac{q}{c d}\right)^{k} \\
& \equiv[n] S_{q}(a, n) \sum_{k=0}^{(n-1) / 2} \frac{\left(a q, q / a, q / c d, q ; q^{2}\right)_{k}}{\left(q^{2} / c, q^{2} / d ; q^{2}\right)_{k}\left(q^{2} ; q^{2}\right)_{k}^{2}} q^{2 k}
\end{aligned}
$$

where

$$
S_{q}(a, n)=\frac{\left(1-q^{n}\right)\left(1+a^{2}-a-a q^{n}\right)}{(1-a)^{2}} q^{(1-n) / 2}-\frac{\left(1-a q^{n}\right)\left(a-q^{n}\right)}{(1-a)^{2}} \frac{\left(q ; q^{2}\right)_{(n-1) / 2}^{2}}{\left(a q^{2}, q^{2} / a ; q^{2}\right)_{(n-1) / 2}} .
$$

Two formulas due to Guo [4, Lemma 2.1] can be stated as

$$
\begin{aligned}
& \left(a q^{2}, q^{2} / a ; q^{2}\right)_{(n-1) / 2} \equiv(-1)^{(n-1) / 2} \frac{\left(1-a^{n}\right) q^{-(n-1)^{2} / 4}}{(1-a) a^{(n-1) / 2}} \quad\left(\bmod \Phi_{n}(q)\right), \\
& \left(a q, q / a ; q^{2}\right)_{(n-1) / 2} \equiv(-1)^{(n-1) / 2} \frac{\left(1-a^{n}\right) q^{\left(1-n^{2}\right) / 4}}{(1-a) a^{(n-1) / 2}} \quad\left(\bmod \Phi_{n}(q)\right),
\end{aligned}
$$

from which we deduce that

$$
\frac{\left(q ; q^{2}\right)_{(n-1) / 2}^{2}}{\left(a q^{2}, q^{2} / a ; q^{2}\right)_{(n-1) / 2}} \equiv \frac{n(1-a) a^{(n-1) / 2}}{\left(1-a^{n}\right) q^{(n-1) / 2}} \quad\left(\bmod \Phi_{n}(q)\right)
$$

and so

$$
[n] S_{q}(a, n) \equiv[n] \Omega_{q}(a, n) \quad\left(\bmod \Phi_{n}(q)^{2}\left(1-a q^{n}\right)\left(a-q^{n}\right)\right) .
$$

Using (4.4) and (4.5), we gain the desired result.

Proof of Theorem 1.1. By L'Hôspital's rule, we have

$$
\begin{aligned}
& \lim _{a \rightarrow 1} \frac{\left(1-a q^{n}\right)\left(a-q^{n}\right)}{(1-a)^{2}}\left\{1-\frac{n(1-a) a^{(n-1) / 2}}{1-a^{n}}\right\} \\
& =\lim _{a \rightarrow 1} \frac{\left(1-a q^{n}\right)\left(a-q^{n}\right)\left\{1-a^{n}-n(1-a) a^{(n-1) / 2}\right\}}{(1-a)^{2}\left(1-a^{n}\right)} \\
& =[n]^{2} \frac{\left(n^{2}-1\right)(1-q)^{2}}{24} .
\end{aligned}
$$

Letting $a \rightarrow 1$ in Theorem 4.2 and utilizing the above limit, we arrive at (1.17) through (2.1) and Lemma 3.3. 
On the basis of numerical calculations, we would like to put forward the following conjecture.

Conjecture 4.3. Let $n$ be a positive integer with $n \equiv 3(\bmod 4)$. Then

$$
\sum_{k=0}^{M}(-1)^{k}[4 k+1] \frac{\left(q ; q^{2}\right)_{k}^{4}\left(q^{2} ; q^{4}\right)_{k}}{\left(q^{2} ; q^{2}\right)_{k}^{4}\left(q^{4} ; q^{4}\right)_{k}} q^{k} \equiv[n]^{2} \frac{\left(q^{3} ; q^{4}\right)_{(n-1) / 2}}{\left(q^{5} ; q^{4}\right)_{(n-1) / 2}} q^{(1-n) / 2} \quad\left(\bmod [n] \Phi_{n}(q)^{4}\right)
$$

where $M=(n-1) / 2$ or $n-1$. In particular, for $p \equiv 3(\bmod 4)$,

$$
\sum_{k=0}^{\left(p^{r}-1\right) / 2}(-1)^{k}(4 k+1) \frac{\left(\frac{1}{2}\right)_{k}^{5}}{k !^{5}} \equiv p^{2 r} \frac{\left(\frac{3}{4}\right)_{\left(p^{r}-1\right) / 2}}{\left(\frac{5}{4}\right)_{\left(p^{r}-1\right) / 2}} \quad\left(\bmod p^{r+4}\right)
$$

\section{References}

[1] S. Ahlgren, K. Ono, Gaussian hypergeometric series evaluation and Apéry number congruences, J. Reine Angew. Math. 518 (2000), 187-212.

[2] T. Kilbourn, An extension of the Apéry number supercongruence, Acta Arith. 123 (2006), $335-348$.

[3] G. Gasper, M. Rahman, Basic Hypergeometric Series (2nd edition), Cambridge University Press, Cambridge, 2004.

[4] V.J.W. Guo, $q$-Supercongruences modulo the fourth power of a cyclotomic polynomial via creative microscoping, preprint, 2019, arXiv:1912.00765.

[5] V.J.W. Guo, Proof of a generalization of the (B.2) supercongruence of Van Hamme through a q-microscope, Adv. Appl. Math. 116 (2020), Art. 102016.

[6] V.J.W. Guo, q-Analogues of Dwork-type supercongruences, J. Math. Anal. Appl. 487 (2020), Art. 124022.

[7] V.J.W. Guo, A $q$-analogue of the (A.2) supercongruence of Van Hamme for primes $p \equiv$ $1(\bmod 4)$, Rev. R. Acad. Cienc. Exactas Fís. Nat. Ser. A Mat. RACSAM 114 (2020), Art. 123.

[8] V.J.W. Guo, A further $q$-analogue of Van Hamme's (H.2) supercongruence for primes $p \equiv 3$ $(\bmod 4)$, Int. J. Number Theory, to appear.

[9] V.J.W. Guo and M.J. Schlosser, A family of $q$-hypergeometric congruences modulo the fourth power of a cyclotomic polynomial, Israel J. Math., to appear.

[10] V.J.W. Guo, M.J. Schlosser, Some $q$-supercongruences from transformation formulas for basic hypergeometric series, Constr. Approx., to appear.

[11] V.J.W. Guo, S.-D. Wang, Some congruences involving fourth powers of central $q$-binomial coecients, Proc. Roy. Soc. Edinburgh Sect. A 150 (2020), 1127-1138.

[12] V.J.W. Guo, W. Zudilin, A q-microscope for supercongruences, Adv. Math. 346 (2019), 329-358.

[13] V.J.W. Guo, W. Zudilin, On a q-deformation of modular forms, J. Math. Anal Appl. 475 (2019), 1636-1646. 
[14] J-C. Liu, On Van Hamme's (A.2) and (H.2) supercongruences, J. Math. Anal. Appl. 471 (2019), 613-622.

[15] J.-C. Liu, F. Petrov, Congruences on sums of $q$-binomial coefficients, Adv. Appl. Math. 116 (2020), Art. 102003.

[16] L. Long, Hypergeometric evaluation identities and supercongruences, Pacific J. Math. 249 (2011), 405-418.

[17] D. McCarthy, R. Osburn, A p-adic analogue of a formula of Ramanujan, Arch. Math. 91 (2008), 492-504.

[18] H.-X. N, H. Pan, On a conjectured $q$-congruence of Guo and Zeng, Int. J. Number Theory 14 (2018), 1699-1707.

[19] H. Swisher, On the supercongruence conjectures of van Hamme, Res. Math. Sci. (2015), $2: 18$.

[20] R. Tauraso, $q$-Analogs of some congruences involving Catalan numbers, Adv. Appl. Math. 48 (2009), 603-614.

[21] L. Van Hamme, Some conjectures concerning partial sums of generalized hypergeometric series, in: p-Adic Functional Analysis (Nijmegen, 1996), Lecture Notes in Pure and Appl. Math. 192, Dekker, New York, 1997, pp. 223-236.

[22] X. Wang, M. Yue, A q-analogue of the (A.2) supercongruence of Van Hamme for any prime $p \equiv 3(\bmod 4)$, Int. J. Number Theory, in press; https://doi.org/10.1142/ S1793042120500694.

[23] X. Wang, M. Yue, Some $q$-supercongruences from Watson's ${ }_{8} \phi_{7}$ transformation formula, Results Math. 75 (2020), Art. 71.

[24] W. Zudilin, Congruences for q-binomial coefficients, Ann. Combin. 23 (2019), 1123-1135. 